\title{
Perioperative pregabalin for reducing pain, analgesic consumption, and anxiety and enhancing sleep quality in elective neurosurgical patients: a prospective, randomized, double-blind, and controlled clinical study
}

\author{
*Nir Shimony, MD, ${ }^{2}$ Uri Amit, BPharm, ${ }^{1}$ Bella Minz, MD, ${ }^{2}$ Rachel Grossman, MD, ${ }^{2}$ \\ Marc A. Dany, MD, ${ }^{1}$ Lior Gonen, MD, ${ }^{2}$ Karina Kandov, RN, BSN, ${ }^{2}$ Zvi Ram, MD, ${ }^{2}$ and \\ Avi A. Weinbroum, MD'
}

Departments of ${ }^{1}$ Anesthesia and Post-Anesthesia Care Unit and ${ }^{2}$ Neurosurgery, Tel Aviv Sourasky Medical Center and the Sackler Faculty of Medicine at the Tel Aviv University, Tel Aviv, Israel

\begin{abstract}
OBJECTIVE The aim of this study was to assess in-hospital (immediate) postoperative pain scores and analgesic consumption (primary goals) and preoperative anxiety and sleep quality (secondary goals) in patients who underwent craniotomy and were treated with pregabalin (PGL). Whenever possible, out-of-hospital pain scores and analgesics usage data were obtained as well.

METHODS This prospective, randomized, double-blind and controlled study was conducted in consenting patients who underwent elective craniotomy for brain tumor resection at Tel Aviv Medical Center between 2012 and 2014. Patients received either $150 \mathrm{mg} \mathrm{PGL}(n=50)$ or $500 \mathrm{mg}$ starch (placebo; $n=50$ ) on the evening before surgery, 1.5 hours before surgery, and twice daily for 72 hours following surgery. All patients spent the night before surgery in the hospital, and no other premedication was administered. Opioids and nonsteroidal antiinflammatory drugs were used for pain, which was self-rated by means of a numerical rating scale (score range $0-10$ ).
\end{abstract}

RESULTS Eighty-eight patients completed the study. Data on the American Society of Anesthesiologists class, age, body weight, duration of surgery, and intraoperative drugs were similar for both groups. The pain scores during postoperative Days 0 to 2 were significantly lower in the PGL group than in the placebo group $(p<0.01)$. Analgesic consumption was also lower in the PGL group, both immediately and 1 month after surgery. There were fewer requests for antiemetics in the PGL group, and the rate of postoperative nausea and vomiting was lower. The preoperative anxiety level and the quality of sleep were significantly better in the PGL group $(p<0.01)$. There were no PGL-associated major adverse events.

CONCLUSIONS Perioperative use of twice-daily $150 \mathrm{mg}$ pregabalin attenuates preoperative anxiety, improves sleep quality, and reduces postoperative pain scores and analgesic usage without increasing the rate of adverse effects.

Clinical trial registration no.: NCT01612832 (clinicaltrials.gov)

http://thejns.org/doi/abs/10.3171/2015.10.JNS151516

KEY WORDS craniotomy; pregabalin; perioperative; pain; analgesia; anxiety

$\mathrm{T}$ He most frequently used drugs for the purpose of reducing acute pain are opioids. The introduction of new opioids and surgery-oriented treatment protocols has, however, failed to adequately handle pain. ${ }^{5,6,12}$ Moreover, increased opioid doses and increased rates of their administration may lead to opioid hyperalgesia instead of appropriate analgesia. Multimodal analgesia, i.e., the combination of opioids and nonopioid oral adjuvants, has proven beneficial in preventing such undesired occurrences when regional analgesia is impracticable. $7,20,27,29,45,46,49$ Morphine adjuvants are compounds that have medium-low analgesic potency when used alone. However, when used multimodally, they potentiate the opioids' effects, thus enabling the use of lower opioid dos-

ABBREVIATIONS ASA = American Society of Anesthesiologists; LOS = length of stay; MOE = morphine equivalent; NRS = numerical rating scale; NSAID = nonsteroidal antiinflammatory drug; $\mathrm{PACU}=$ postanesthesia care unit; $\mathrm{PGL}=$ pregabalin; $\mathrm{POD}=$ postoperative day; $\mathrm{PONV}=$ postoperative nausea and vomiting. SUBMITTED June 30, 2015. ACCEPTED October 5, 2015. INCLUDE WHEN CITING Published online February 12, 2016; DOI: 10.3171/2015.10.JNS151516.

* Dr. Shimony and Mr. Amit contributed equally to this work. 
es and limiting their side effects along with the achievement of optimal analgesia status and satisfaction rates. ${ }^{9}$ One group of newer products that are not analgesics per se but can act as co-adjuvant opioids are gabapentinoids.

One gabapentinoid-pregabalin (PGL; Lyrica) -is an antiepileptic drug that has been shown to expedite the analgesic effects of other drugs for diabetic neuropathy, postherpetic neuralgia, and neuropathic pain. ${ }^{18,31}$ PGL binds selectively to the $\mathrm{Ca}(\mathrm{V}) \alpha_{2} \delta$ auxiliary subunit of presynaptic voltage-gated calcium channels, thus decreasing the release of pro-nociceptive neuropeptides, including glutamate, noradrenaline, and substance $\mathrm{P}{ }^{42}$ This inhibition prevents the release of the excitatory neurotransmitters that activate pain pathways. ${ }^{28}$ Most of the reviews and meta-analyses of PGL (and its structural analog gabapentin) have shown that their perioperative use permits an opioid-sparing effect and improves postoperative pain scores in comparison with placebo. 3,17,21,24,34,47 However, adverse effects, such as visual disturbance, sedation, dizziness, and headache, may occur, especially at higher doses. Pregabalin had initially been tested in relatively less painful surgical procedures, such as laparoscopic, gynecological, and minimally invasive orthopedic surgeries, as well as other ambulatory surgeries. ${ }^{1}$ Recently, it has been tested in more painful surgical procedures such as lumbar spine surgery and knee arthroplasty. ${ }^{8,10}$

Pain after an infratentorial procedure is common and at times it is severe. ${ }^{39,44}$ One study in which the majority of the procedures were supratentorial noted that $56 \%$ of the patients reported persistent pain even at 2 months after surgery and $50 \%$ experienced neuropathic symptoms. ${ }^{2}$ Thus, intracranial surgery induces meaningful postoperative pain, and proper treatment is essential.

In neurosurgical patients, the rapid and sustained regaining of consciousness, as well as pain control that minimally affects orientation and lucidity, are preferred. The anesthesia protocol should also exclude opioids due to their side effects, such as sedation, meiosis, nausea, and vomiting, allowing the prompt recognition of incipient intracranial catastrophe and not interfering with intracranial physiology. There is also a concern about the respiratory depressant effects of opioids that can increase circulating carbon dioxide tension, which provokes changes in cerebral blood flow and intracranial pressure. ${ }^{16}$

Although as an anticonvulsant PGL may minimally delay emergence from anesthesia ${ }^{43}$ it remains unknown if its prolonged perioperative administration would interfere with patient safety and outcomes after intracranial surgery.

The objectives of this study were to assess if there are beneficial preemptive and preventive effects of PGL on 1) the immediate (0-3 days) postoperative analgesic requirements and pain scores (primary goals) and whenever available the late (up to 3 months) self-assessed pain levels and drug usage (primary goals) and 2) presurgical anxiety levels and quality of sleep (secondary goals) in patients undergoing elective neurosurgical procedures.

\section{Methods}

\section{Patient Definitions}

Patients who were in Classes I to III according to the
American Society of Anesthesiologists (ASA) Physical Status Classification System and scheduled to undergo craniotomy for the removal of a brain tumor were enrolled into this study. This study was approved by the institutional human research and ethics committee of the Tel Aviv Sourasky Medical Center and was registered at clinicaltrials.gov (NCT01612832). All participants were given a full explanation of the study protocol, study drug, and numerical rating scale (NRS) scores during the preanesthesia interview. The patients then signed Helsinkiapproved informed consent. Surgery was performed under either general anesthesia or monitored anesthesia care (i.e., "awake").

\section{Exclusion Criteria}

Patients were excluded if they had a history of allergy to opioids, propofol, PGL, nonsteroidal antiinflammatory drugs (NSAIDs), or dipyrone, chronic pain unrelated to the cause of intervention, psychiatric disorders, or the use of centrally acting antidepressants or antipsychotic drugs, sedatives, or antiepileptics. Patients who were younger than 18 years of age or older than 80 years, in military service, or pregnant were also excluded, as were emergency cases, patients receiving anticonvulsants and/or antiemetics, patients planning to undergo deep brain stimulation procedures, and patients unable to comprehend or sign the consent form. Finally, patients with severe liver or renal failure and those expected to require a long-term postoperative stay in the hospital were excluded from this study.

\section{Dropouts or Drug Interruption}

Changes ( $>20 \%$ ) from baseline in the patient's heart rate, systolic blood pressure (per medical history), abnormal end-tidal carbon dioxide or arterial carbon dioxide partial pressure fluctuations, or peripherally measured fraction of oxygen-saturated hemoglobin $(<92 \%)$ under $40 \%$ oxygen administered via facemask, at 3 consequent time points postoperatively, led to the exclusion of the patient's data from that point onward until these parameters normalized. Also, patients who required postoperative controlled/assisted ventilation for longer than 4 hours or were combative or incoherent while requesting analgesia were excluded from the study from that time until spontaneous ventilation was retained or a stable mental state had been restored. Reintervention within 72 hours also meant the permanent exclusion of patient data from the time of the event. Finally, a patient who was considered by the attending surgeon as being excessively sedated was dropped from the study, and the drug study was stopped.

\section{Study Design}

This prospective study was performed in a placebocontrolled, double-blind (ratio 1:1) manner. Randomization was accomplished using the institution's pharmacyprepared patient list and RANDOM.org's software model (Randomness and Integrity Services Ltd.). Patients in the PGL group received capsules containing $150 \mathrm{mg}$ PGL at 8:00 PM the night before surgery and 1.5 hours before undergoing surgery the next day. Patients in the placebo group were given identical capsules containing $500 \mathrm{mg}$ 
starch at the same time points. No other premedication drugs were given to the patients preoperatively. Postoperatively, they continued to receive their allocated capsules 2 hours after surgery, and then twice daily for the next 72 hours.

The same surgical and anesthesia teams performed all procedures. Surgery consisted of infra- or supratentorial brain tumor/mass resection. General anesthesia consisted of propofol and a muscle relaxant, fentanyl boluses, or remifentanil by infusion, as deemed adequate by the anesthesiologist, as hemodynamically permitted, and as surgically required. Oxygen and isoflurane (infrequent) were provided, while no nitrous oxide was administered.

Prior to surgical incision, $20 \mathrm{ml}$ lidocaine $(1.5 \%)$ plus bupivacaine $(0.25 \%)$ was injected into the subcutaneous and deeper areas at the surgical site. In the cases of awake surgery (i.e., monitored anesthesia care), propofol, fentan$\mathrm{yl}$, or remifentanil was used to maintain minimal hypnosis and analgesia and avoid apnea, permitting the patient's cooperation at intervals either for motor or speech tasks (such as verb generation, free speech, or naming) that were performed by a dedicated advanced neuropsychologist. After the surgical procedure, boluses of morphine and propofol were available to the patient, as deemed necessary by the attending anesthesiologist, in order to keep the patient comfortable and alert but without hemodynamic fluctuations.

After the patient was fully conscious, had stable vital signs, was extubated and breathed spontaneously, and could carry out basic commands, he/she was transferred to the postanesthesia care unit (PACU) where the first vital signs were registered. When the patient was observed as being coherent, and upon the demand for analgesia, intravenous paracetamol or $1000 \mathrm{mg}$ dipyrone was administered by the attending nurse. Further requests for pain relief were satisfied with intramuscular $75 \mathrm{mg}$ diclofenac or $100 \mathrm{mg}$ tramadol by slow intravenous infusion (not routinely accompanied by an antiemetic agent). If all the above analgesics were ineffective, $1 \mathrm{mg}$ intravenous morphine was given until the NRS score was $\leq 5$ of $10 .{ }^{35}$ Ten milligrams intravenous metoclopramide was the first drug of choice for postoperative nausea and vomiting (PONV), followed by a slow administration of $3 \mathrm{mg}$ granisetron if still experiencing PONV. Four milligrams intravenous ondansetron was subsequently administered for persistent PONV.

The patients remained in the PACU for a minimum of 3 hours to ensure the recognition of late-onset pain, sedation rebound, and neurological deterioration. They were then transferred to the neurosurgical intensive care unit or to the neurosurgical ward according to the surgeon's instructions.

\section{Efficacy Measurements}

Various parameters were assessed perioperatively by the attending nurses. In addition to vital signs and level of consciousness, the following 6 patient-rated and objective parameters were assessed, first hourly and then every 8 hours: 1$)$ subjectively rated pain intensity using a 0 to 10 NRS ( $0=$ no pain; $10=$ unbearable pain). 2 ) Perioperative opioid consumption. The morphine equivalent (MOE) was calculated based on commonly used conversion tables of morphine. Daily or total MOE is expressed as follows: $\mathrm{MOE}=$ oral morphine $\mathrm{mg}$ dose + oral Percocet $\mathrm{mg} \times 1.5$ + oral Oxycod Syrup mg $\times 2+$ oral tramadol mg/10 + intravenous morphine $\mathrm{mg} \times 3+$ intravenous tramadol $\mathrm{mg} / 3.33$ (as the intravenous potency is $1 / 10$ of intravenous morphine and $\times 3$ for oral tramadol). ${ }^{32,40,41} 3$ ) Postoperative rates of drug usage, including analgesics and antiemetics. 4) Length of stay (LOS) in the hospital. 5) Patient overall satisfaction rate (by telephone survey). 6) Self-rated (NRS) pain scores and analgesics usage at 2 weeks and 1 and 3 months following surgery (by telephone survey, whenever obtainable).

Subjectively rated anxiety was assessed on a 0 to 10 NRS $(0=$ very calm; $10=$ very anxious). This parameter was evaluated preoperatively during enrollment and immediately prior to surgery. Subjectively rated quality of sleep, on a 0 to 10 NRS ( $0=$ unable to sleep because of anxiety; $10=$ deep and satisfactory sleep), was assessed immediately prior to surgery.

\section{Safety Assurance}

Throughout the study, any complaint of untoward effect was noted by the attending nurse or physician and treated appropriately. Several undesired effects that had been previously reported ${ }^{10}$ were especially documented, including dizziness, dry mouth, peripheral edema, headache, sedation, blurred vision, PONV, and pruritus.

\section{Data Analyses}

The analyses were performed using SPSS for Windows (version 21, IBM). Based on our previous studies, ${ }^{29}$ a power table in which delta (the mean 6-hour difference in pain rated by NRS) was determined as $1.5 \pm 1.0$, alpha $=0.05$, and a power of 0.95 resulted in 35 patients/group. Data on age, weight, ASA class, duration of surgery, levels of preoperative anxiety, quality of sleep, patient overall satisfaction, dosages of intraoperative drugs, and total or per patient dosages of perioperative analgesics were all compared using the 2-tailed t-test. Analysis of the parameters whose values fell in large ranges was verified using the Mann-Whitney U-test. The patients' short- and longterm self-rated pain scores, in- and out-of-hospital drug usages, and in-hospital LOS were analyzed in a similar manner. The sex distribution was analyzed using the chisquare test. The number of times the patients requested anti-nociceptives, and the rate of side effects attributed to the tested drugs, were analyzed using the Fisher exact test. All values are expressed as the mean \pm standard deviation, with statistical significance being defined as $\mathrm{p} \leq 0.05$.

\section{Results \\ Demographics}

Eighty-eight of the 100 enrolled patients completed the study (Fig. 1). There were no statistically significant differences between the groups with regard to age, weight, sex distribution, ASA class, preoperative steroid administration, LOS, rates of satisfaction and 30-day reoperation, side effects, and pain and anxiety levels at the time of admission (Tables 1-6). The duration of surgery and the administered anesthetic and analgesic drugs (Table 2) were also similar; all patients woke up immediately upon 


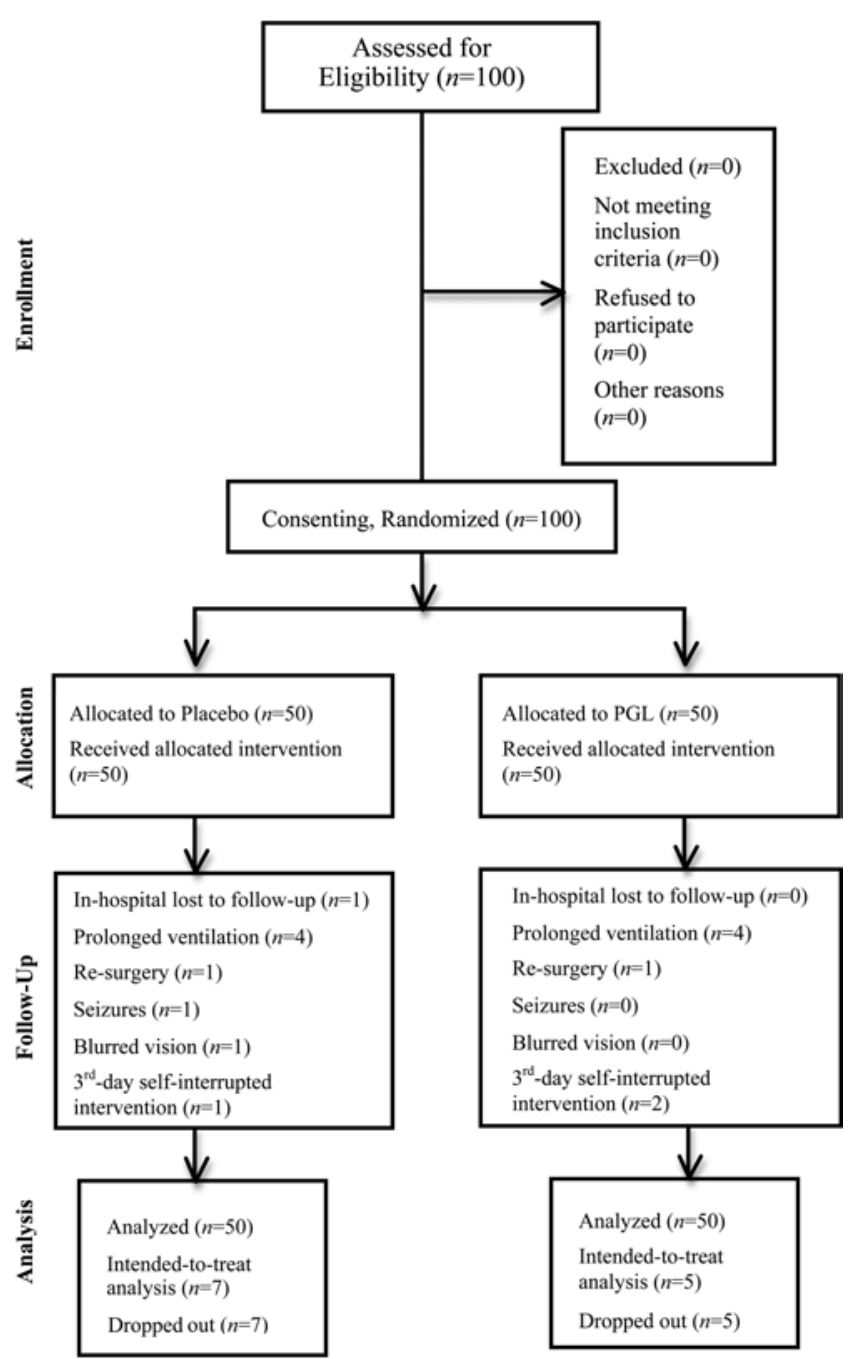

FIG. 1. Study CONSORT flowchart.

the interruption of anesthetics and were fully conscious. The number of patients who required blood replacement and their amounts, and the amounts of fluids infused during surgery, were within the normal ranges for the specific interventions and were similar between the 2 groups (data not shown).

\section{Pain Scores and Analgesics Use}

The 2 primary goals of this investigation were to assess the immediate ( 0 - to 3 -day) postoperative pain scores and analgesic consumption, the late (up to 3-month) pain levels, and the use of painkillers outside the hospital. Importantly, the preoperative pain scores were similar among study individuals (Table 3); only 2 patients in the placebo group and 1 patient in the PGL group were using NSAIDs preoperatively with optimal pain control. The self-rated pain scores $(0-10)$ that were recorded during the period of stay in the PACU were 33\% lower in the PGL group, although the difference did not reach a level of significance. During the rest of the day of surgery (postoperative day [POD] 0), as well as POD 1, the PGL patients rated their levels of pain as being significantly lower than those reported by the placebo patients. The self-rated pain levels
TABLE 1. Demographic data*

\begin{tabular}{lccc}
\hline \multicolumn{1}{c}{ Parameter } & Pregabalin & Placebo & p Value \\
\hline Mean age, yrs & $52.6 \pm 15.5$ & $51.6 \pm 16.8$ & 0.75 \\
\hline Sex & & & \\
\hline$\%$ male & 42 & 55 & 0.20 \\
\hline$\quad 48$ & 45 & 0.55 \\
\hline Mean weight, $\mathrm{kg}$ & $76.2 \pm 15.5$ & $79.2 \pm 22$ & 0.44 \\
\hline Mean ASA class & $2.05 \pm 0.7$ & $2.21 \pm 0.53$ & 0.87 \\
\hline $\begin{array}{l}\text { Mean preop steroid treatment, } \\
\text { mg/patient }\end{array}$ & $5.68 \pm 3.28$ & $6.67 \pm 3.67$ & 0.36 \\
\hline $\begin{array}{l}\text { No. of preop steroid users } \\
\text { * Mean values are presented as the mean } \pm \text { SD. }\end{array}$ & & \\
\hline
\end{tabular}

continued to be lower $(\sim 10 \%)$ in the PGL group on PODs 2 and 3, but these differences did not reach a level of significance (Table 3). During the following days, the PGL pain scores were almost equal to those of the placebo pain scores (data not shown).

Overall, the PGL patients requested considerably fewer analgesics of any type in comparison with the placebotreated controls (Table 3). The PGL patients received 33\% fewer opioids, and their overall opioid consumption was minimal in the neurosurgical ICU (Table 3). After being discharged from the PACU, on PODs 0 to 2, the total amounts and fractions of drug doses per user, as well as the rates of usage for all types of analgesics, were constantly and significantly lower among the PGL patients than in controls. The amounts of drugs and the number of doses requested on POD 3 were consistently lower among the PGL patients in comparison with the controls; however, these differences did not reach a level of significance because of the small number of individuals requesting them (Type II statistical error).

The late (out-of-hospital) pain scores and the rates of analgesic use (Table 4) indicated that the pain scores of the PGL patients were $16 \%$ to $43 \%$ lower than those of controls although not reaching statistical significances (Type II statistical error). Painkillers were used similarly by both groups; however, 33\% fewer PGL patients used analgesics

TABLE 2. Surgery and intraoperative anesthesia data*

\begin{tabular}{lccc}
\hline \multicolumn{1}{c}{ Parameter } & Pregabalin & Placebo & p Value \\
\hline Anesthesia & & & \\
\hline$\quad$ MAC & 8 & 8 & 1.0 \\
\hline$\quad$ General & 42 & 42 & 1.0 \\
\hline Mean duration of surgery, hrs & $5.37 \pm 2.17$ & $5.31 \pm 2.26$ & 0.89 \\
\hline Surgical approach & & & \\
\hline$\quad$ Supratentorial & 6 & 7 & 1.0 \\
\hline$\quad$ Infratentorial & 44 & 43 & 1.0 \\
\hline Mean fentanyl, mg/patient & $0.26 \pm 0.13$ & $0.34 \pm 0.44$ & 0.24 \\
\hline Mean morphine, mg/patient & $6.1 \pm 3.31$ & $5.34 \pm 2.35$ & 0.26 \\
\hline Mean remifentanil, mg/patient & $2.81 \pm 1.83$ & $2.7 \pm 2.2$ & 0.81 \\
\hline Mean propofol, mg/patient & $1191 \pm 1118$ & $1306 \pm 1153$ & 0.59 \\
\hline
\end{tabular}

$\mathrm{MAC}=$ monitored anesthesia care

* Values are number of patients unless otherwise noted. Mean values are presented as mean \pm SD. 
TABLE 3. In-hospital data*

\begin{tabular}{|c|c|c|c|c|}
\hline Time Point & Parameter & Pregabalin & Placebo & $p$ Value \\
\hline On admission & Pain, mean NRS score & $0.36 \pm 1.21$ & $0.54 \pm 1.54$ & 0.30 \\
\hline Preop & Pain, mean NRS score & $0.42 \pm 1.48$ & $0.45 \pm 1.47$ & 0.81 \\
\hline \multirow[t]{6}{*}{ PACU } & Pain, mean NRS score & $0.96 \pm 1.22$ & $1.28 \pm 1.33$ & 0.16 \\
\hline & MOE, mean mg/user & $17.96 \pm 13.96$ & $23.48 \pm 21.05$ & 0.33 \\
\hline & MOE, no. of users & 26 & 30 & 0.41 \\
\hline & Non-opioids, mean doses/user† & $1.26 \pm 0.48$ & $1.32 \pm 0.61$ & 0.92 \\
\hline & Non-opioids, no. of users† & 13 & 13 & 1.0 \\
\hline & All analgesics, no. of users & 29 & 34 & 0.42 \\
\hline \multirow[t]{7}{*}{ Neurosurgical ICU } & Mean LOS, days & $2.43 \pm 4.19$ & $2.3 \pm 4.46$ & 0.97 \\
\hline & Pain, mean NRS score & $1.56 \pm 1.95$ & $2.92 \pm 2.05$ & $<0.01$ \\
\hline & MOE, mg/user & 0 & $0.76 \pm 3.31$ & 0.11 \\
\hline & MOE, no. of users & 0 & 3 & 0.12 \\
\hline & Non-opioids, mean doses/user† & $1.82 \pm 0.72$ & $1.74 \pm 0.92$ & 0.76 \\
\hline & Non-opioids, no. of users & 17 & 26 & 0.05 \\
\hline & All analgesic, no. of users & 17 & 30 & $<0.01$ \\
\hline \multirow[t]{9}{*}{ POD 1} & Pain, mean NRS score & $1.5 \pm 1.71$ & $2.26 \pm 1.59$ & $<0.01$ \\
\hline & MOE, mg/user & $7.5 \pm 0 \ddagger$ & $9.46 \pm 4.46$ & 0.74 \\
\hline & MOE, no. of users & 1 & 12 & $<0.01$ \\
\hline & Non-opioids, mean doses/user† & $1.64 \pm 0.87$ & $1.88 \pm 1.01$ & 0.34 \\
\hline & Non-opioids, no. of users & 25 & 31 & 0.01 \\
\hline & Diclofenac, no. of users & 2 & 11 & $<0.01$ \\
\hline & Dipyrone, mean doses/user & $1.39 \pm 0.62$ & $1.78 \pm 0.82$ & 0.08 \\
\hline & Dipyrone, no. of users & 22 & 25 & 0.61 \\
\hline & All analgesic, no. of users & 26 & 34 & 0.02 \\
\hline \multirow[t]{7}{*}{ POD 2} & Pain, mean NRS score & $1.27 \pm 1.61$ & $1.47 \pm 1.56$ & 0.32 \\
\hline & MOE, mean mg/user & $15 \pm 0 \ddagger$ & $6.3 \pm 1.68$ & 0.12 \\
\hline & MOE, no. of users & 1 & 5 & 0.05 \\
\hline & Non-opioids, mean doses/user† & $1.38 \pm 0.62$ & $1.73 \pm 0.82$ & 0.15 \\
\hline & Non-opioids, no. of users & 16 & 26 & 0.04 \\
\hline & Dipyrone, no. of users & 13 & 24 & 0.03 \\
\hline & All analgesics, no. of users & 16 & 26 & 0.03 \\
\hline \multirow[t]{6}{*}{ POD 3} & Pain, mean NRS score & $1.11 \pm 1.43$ & $1.13 \pm 1.39$ & 0.69 \\
\hline & MOE, mean mg/user & $7.5 \pm 0 \ddagger$ & $15 \pm 0$ & 0.16 \\
\hline & MOE, no. of users & 1 & 2 & 1.0 \\
\hline & Non-opioids, mean doses/user† & $1.5 \pm 0.67$ & $1.75 \pm 0.93$ & 0.42 \\
\hline & Non-opioids, no. of users & 12 & 16 & 0.34 \\
\hline & All analgesics, no. of users & 12 & 16 & 0.23 \\
\hline \multirow[t]{2}{*}{ Discharge } & Pain at discharge, mean NRS score & $0.68 \pm 1.5$ & $0.98 \pm 2.11$ & 0.79 \\
\hline & Dipyrone, no. of requests & 4 & 5 & 1.0 \\
\hline \multirow[t]{5}{*}{ Other } & Mean in-hospital LOS, days & $8.3 \pm 7.77$ & $7.9 \pm 8.64$ & 0.77 \\
\hline & 30-day complications, $\%$ of cohort & 10 & 8 & 0.74 \\
\hline & 30 -day re-surgery, $\%$ of cohort & 4 & 4 & 1.0 \\
\hline & No. of dropouts§ & 5 & 7 & 0.25 \\
\hline & No. of drug-attributed adverse events & Dizziness 2 , sedation 1 & Dizziness 0 , sedation 2 & 0.55 \\
\hline
\end{tabular}

\footnotetext{
* Values are shown as the mean \pm SD, percent of cohort, or number of patients. Boldface type indicates statistical significance.

$\dagger$ Doses of any analgesic or all combined.

$\ddagger$ One dose only.

$\S$ Dropout specifications (also see text and Fig. 1).
} 
TABLE 4. Long-term follow-up (27 patients/group)*

\begin{tabular}{clccr}
\hline Time Point & \multicolumn{1}{c}{ Parameter } & Pregabalin & Placebo & $p$ Value \\
\hline \multirow{2}{*}{2 wks } & Pain, mean NRS score & $1.68 \pm 2.71$ & $2.93 \pm 3.44$ & 0.19 \\
\hline \multirow{2}{*}{$1 \mathrm{mo}$} & Pain, mean NRS score & $1.46 \pm 2.2$ & $2.28 \pm 3.08$ & 0.51 \\
\cline { 2 - 5 } & Drug usage & $48 \%$ & $63 \%$ & $<0.01$ \\
\hline \multirow{2}{*}{3 mos } & Pain, mean NRS score & $1.28 \pm 2.16$ & $1.51 \pm 2.8$ & 0.72 \\
\cline { 2 - 5 } & Drug usage & $27 \%$ & $34 \%$ & 0.12 \\
\cline { 2 - 5 } & Overall satisfaction rate, mean NRS score & $8.0 \pm 1.93$ & $8.5 \pm 1.84$ & 0.48 \\
\hline
\end{tabular}

* Values are shown as the mean \pm SD or percent of cohort. Boldface type indicates statistical significance.

during the 1st postoperative month compared with the placebos (Table 4).

\section{PGL, Presurgical Anxiety, and Sleep Quality}

The 2 secondary goals of this study were to assess the presurgical level of anxiety and the associated disturbances of sleep. The levels of anxiety at the time of recruitment were similarly high in both groups (Table 5; Fig. 2). When interviewed prior to surgery, the PGL patients had a selfrated anxiety level that was significantly lower than that of the controls. The quality of sleep, which was also assessed before entering the operating room, was rated significantly better by the PGL patients (i.e., a higher NRS score) than the controls (Table 5; Fig. 3). These figures represented the effect of 2 doses and 1 dose of PGL, respectively.

\section{PONV and the Use of Antiemetics}

Table 6 lists the use of antiemetics during the patients' hospital stays and the number of cases of spontaneous vomiting. Antiemetics were administered intraoperatively as deemed necessary-but not prophylactically-by the attending anesthesiologist, and the number of doses administered in each group were almost identical. Postoperatively, antiemetics were given when a patient complained of sustained nausea or if vomiting occurred. Antiemetics were administered for those reasons to 34 PGL patients compared with 47 controls $(p=0.0092)$. The total rate of vomiting in the PGL group during the hospital stay was $40 \%$ that of the control group ( $p=0.034$; Table 6$)$.

\section{Overall Satisfaction, Adverse Events, and Complication Rates}

The overall satisfaction rates with regard to perioperative experience were similarly high in both groups (Table 4). The number of adverse events in the PGL group was similar to that in the control group (Table 3 ). The low rates of surgical complications and reoperation in both groups did not differ from the values in the institutional database records (data not shown).
Finally, the dropout rates (5 PGL vs 7 placebo patients) overlapped with the cases of self-drug interruption, nondrug causes, and drug-associated reasons (3 patients in the PGL group vs 2 patients in the placebo group) and were almost identical in both groups (Fig. 1; Table 3). The sedation cases were excluded from the study by the surgeon on POD 2 from the time of their identification, and the drugs were stopped (excessive sedation was later discussed and its correlation to PGL remained unsettled).

\section{Discussion}

Morphine and morphine-like agonists are the cornerstone of perioperative analgesia. The use of opioids is often disputed by neurosurgeons because of their association with adverse events. Such sequelae can be minimized by the addition of nonopioid adjuvants. ${ }^{20,35,37,45}$ This randomized, controlled, double-blind trial demonstrates-for what we believe to be the first time-that the addition of twice-daily $150 \mathrm{mg}$ PGL to opioids and nonopioids for 3 perioperative days was safe and beneficial for patients who underwent elective intracranial surgery. PGL reduced the self-rated pain scores and analgesic requirements by up to $33 \%$ during PODs 0 to 2 . Furthermore, the patients' preoperative quality of sleep significantly improved, and their levels of anxiety were lowered by 1 and 2 doses of PGL, respectively. PGL use following the described protocol did not produce either excessive sedation or any other adverse effects but did reduce the PONV rates and the number of requests for antiemetics.

Opioids are the main analgesics used for treating moderate to severe pain when regional anesthesia is inapplicable. Surgical intervention within the brain requires particular attention since the prompt regaining of consciousness, responsiveness to commands, and stable hemodynamic and respiratory conditions are crucial during the entire postoperative period. Some surgeons also reject the use of NSAIDs due to the risk of intracerebral rebleeding, while others raise concerns about the depressive effects of multiple doses of opioids and the consequent increase in ce-

TABLE 5. Immediate preoperative anxiety level and sleep quality ${ }^{*}$

\begin{tabular}{ccccc}
\hline Time Point & Parameter & Pregabalin & Placebo & p Value \\
\hline On admission & Anxiety level, mean NRS score & $4.92 \pm 2.95$ & $5.62 \pm 2.71$ & 0.28 \\
\hline Preoperatively & Anxiety level, mean NRS score & $3.13 \pm 2.3$ & $4.25 \pm 2.65$ & 0.04 \\
\cline { 2 - 5 } & Sleep quality, mean NRS score & $7.58 \pm 2.40$ & $4.57 \pm 3.10$ & $<0.01$ \\
\hline
\end{tabular}

* Values are mean \pm SD. Boldface type indicates statistical significance. 
TABLE 6. Antiemetics usage*

\begin{tabular}{llccc}
\hline \multicolumn{1}{c}{ Time Point } & \multicolumn{1}{c}{ Parameter } & Pregabalin & Placebo & $p$ Value \\
\hline \multirow{2}{*}{ Intraop } & Metoclopramide, mean mg/user & $10 \pm 0$ & $10 \pm 0$ & 1.0 \\
& Metoclopramide, no. of users & 4 & 3 & 0.55 \\
\cline { 2 - 5 } & Ondansetron, mean mg/user & $7.09 \pm 2.96$ & $7.52 \pm 1.17$ & 0.56 \\
\cline { 2 - 5 } & Ondansetron, no. of users & 21 & 20 & 0.77 \\
\hline PACU & Metoclopramide, mean mg/user & $10 \pm 0$ & $10.83 \pm 2.88$ & 0.50 \\
\cline { 2 - 5 } & Metoclopramide, no. of users & 6 & 12 & $<0.05$ \\
\cline { 2 - 5 } & Ondansetron, mean mg/user & $5.16 \pm 2.22$ & $4.85 \pm 1.7$ & 0.74 \\
\cline { 2 - 5 } & Ondansetron, no. of users & 6 & 14 & 0.01 \\
\hline All antiemetic, mean doses/user (including granisetron) & $1.16 \pm 0.38$ & $1.21 \pm 0.51$ & 0.77 \\
\hline Neurosurgical ICU & All antiemetic, no. of users (including granisetron) & 12 & 23 & $<0.01$ \\
\hline POD1 & All antiemetics, no. of requests & 4 & 6 & 0.4 \\
\hline POD2 & All antiemetics, no. of requests & 2 & 5 & 0.27 \\
\hline POD3 & All antiemetics, no. of requests & 2 & 1 & 0.31 \\
\hline POD 0-3 & All antiemetics, no. of requests & 1 & 1 & 1.0 \\
\hline
\end{tabular}

* Mean values are mean \pm SD. Boldface type indicates statistical significance.

rebral blood flow and intracranial pressure. Polypharmacy was shown to reduce morphine consumption and its undesired effects by one potentiating the other's analgesic attributes. $4,13,14,28,45,48$

Early PGL dosing had ranged between 300 and 600 $\mathrm{mg} /$ dose, ${ }^{45}$ while currently the most accepted dose is twice-daily $150 \mathrm{mg}$ PGL. ${ }^{17,33,45}$ Premedication with $75 \mathrm{mg}$, followed by the same dose twice daily for 7 postoperative days, reportedly spared opioids meaningfully and reduced pain after breast augmentation. ${ }^{23}$ Preemptive $300 \mathrm{mg}$ PGL administered 1 hour prior to total knee arthroplasty with an additional twice-daily $150 \mathrm{mg}$ PGL for 14 postoperative days also resulted in the lower use of oral opioid consumption and a significantly lower occurrence (5\%-8\%) of chronic knee pain after 3 and 6 months compared with

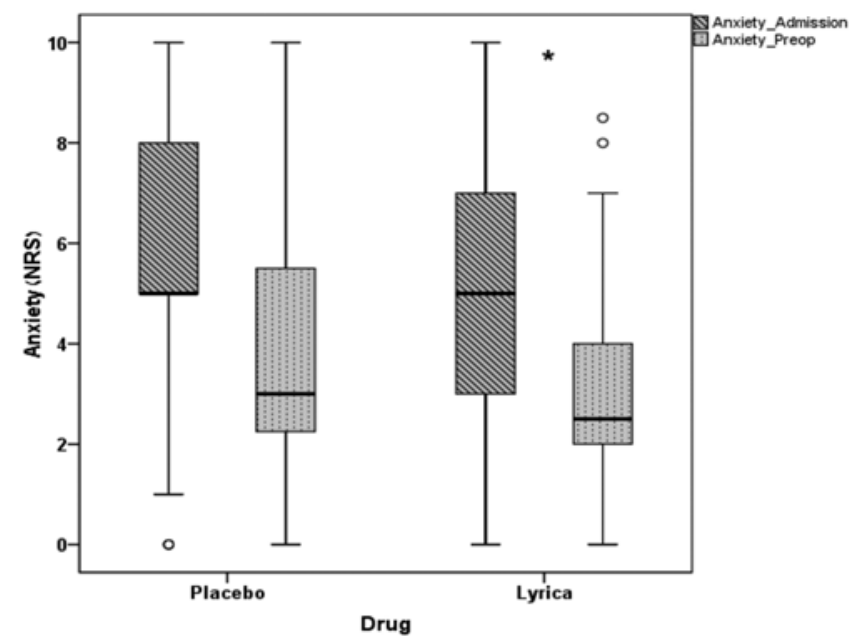

FIG. 2. The patients' preoperative anxiety levels. Anxiety was self-rated by the patients using an NRS $(0-10)$ at both enrollment and after 2 doses of pregabalin just before surgery. *The reduction in anxiety was significant $(p=0.04)$ only in the pregabalin group. The open dots indicate outliers. placebo. ${ }^{10}$ Engelman and Cateloy recently concluded that the lowest daily effective dose of PGL ranges between 225 and $300 \mathrm{mg} .{ }^{21}$ Finally, 8-hour $150 \mathrm{mg}$ PGL in patients scheduled for abdominal hysterectomy or myomectomy led to less morphine consumption during the first 48 hours, while the pain scores at rest and upon coughing following the ingestion of codeine plus acetaminophen (Lonalgal) tablets did not differ from placebo. ${ }^{22}$ Thus, a higher daily dosage does not seem to reduce the use of rescue drugs, ${ }^{22}$ as was documented in the present study as well. One possible explanation for these conflicting trial-and-error dosing results is that the clinical effects of PGL are not solely pharmacologically modulated, but may also depend upon the activities of neurological pathways within the pain centers whose mechanisms are not yet fully understood, e.g., pain neuroadaptation..$^{45}$

The best timing for gabapentinoid administration is

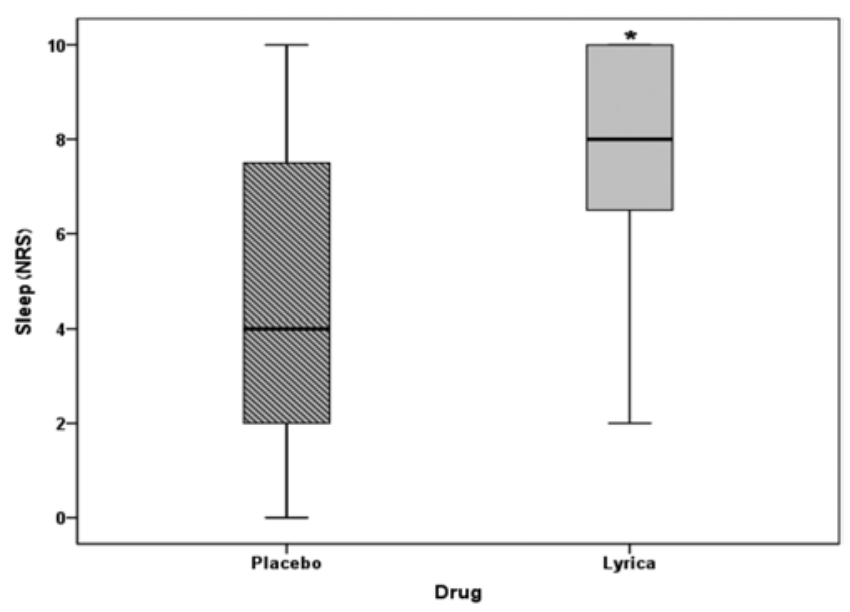

FIG. 3. Preoperative quality of sleep. Sleep quality was self-rated by the patients using NRS $(0-10)$ before surgery and after 1 dose of pregabalin. *The pregabalin patients rated treatment significantly $(p<0.01)$ better than the placebo group. 
well established. A meta-analysis of the effect of preoperative gabapentin on postoperative pain in 12 randomized controlled trials (896 patients) showed that its postoperative analgesic effects were best if administration started within less than 4 hours before surgery. ${ }^{25}$ Pain intensity after administering $75 \mathrm{mg}$ PGL at 1 hour before surgical molar extraction under local anesthesia was higher than if administered after it, but the analgesic consumption and side effects were similar. ${ }^{15}$ Thus, twice-daily PGL optimally affects anti-nociception when administered a few hours before surgery and continued thereafter as described in the current study.

PGL has highly predictable and linear pharmacokinetics, with a profile that makes it easier to use in clinical practice compared to gabapentin. Its absorption is extensive, rapid, and dose-independent: the peak plasma concentration is reached within 1 hour, and a steady state is achieved within 24 to 48 hours of treatment. ${ }^{3}$ It also has dose-proportional maximal plasma concentrations. PGL does not bind to plasma proteins and is excreted virtually unchanged by the kidneys ( $<2 \%$ is metabolized). ${ }^{3,38}$ Dose adjustment may be necessary in patients with renal insufficiency. Due to the lack of hepatic metabolism, induction, or inhibition of liver enzymes, PGL is unlikely to cause, or be subjected to, pharmacokinetic drug-drug interactions. Administering the drug up to 4 hours before surgery ${ }^{25}$ is especially useful if an anti-allodynic effect is to be attained at the start of the postoperative period and assuming surgery lasts approximately 2 hours. ${ }^{11}$ We therefore adhered to these timetables. We also added an extra 2-hour dose of PGL when we expected a longer duration of surgery.

Safety issues in the setting of PGL bear mentioning. Cardiac patients 75 years or older who were treated preoperatively with $150 \mathrm{mg}$ PGL and then twice-daily $75 \mathrm{mg}$ for 5 days reportedly consumed $44 \%$ to $48 \%$ less oxycodone postoperatively on PODs 2 to $5 .{ }^{36}$ They also exhibited less confusion on the 1st postoperative day, although time to extubation was longer. ${ }^{36}$ In our current study, this latter was not an issue, as long as patients woke up calm, hemodynamically stable, coherent, and following basic commands. Importantly, PGL was reportedly associated with dizziness, ataxia, blurred vision, and diplopia in a dose-dependent manner. ${ }^{22,45}$ PGL was also reported to decrease PONV in patients who did not receive anti-PONV prophylaxis, ${ }^{19}$ as observed in the present study as well. We did not observe any increase in dizziness or PGL-associated sedation rates, and our data support an antiemetic effect of PGL. Nevertheless, 4 reviewed studies reported no prevention of PONV. ${ }^{17}$ We found that PGL was associated with fewer cases of PONV, as well as with a reduced use of antiemetics $(74 \%$ for the PGL-treated vs $94 \%$ for our placebo-administered elective neurosurgical patients requested antiemetics).

The patients in this study were administered PGL both before and after the scheduled intervention. Patients suffering from pain in general or being treated for it, using preoperative anticonvulsants or centrally acting drugs (e.g., antiemetics), were excluded, thus limiting confounding factors related to preoperative anxiety and sleep quality herein investigated. PGL acted favorably on those 2 factors, without increasing craniotomy-relevant adverse effects (e.g., dizziness, sedation, or PONV). This is in opposition to a study that demonstrated that preoperative sin- gle-dose PGL increased perioperative sedation in a group treated with $300 \mathrm{mg}$ PGL and failed to reduce preoperative anxiety at any dose ranging between 75 and $300 \mathrm{mg} .{ }^{47}$ However, these 2 elements were not tested postoperatively in our study because of the many confounding issues thereafter, such as pain, anesthetics, and opioid effects on the brain, subtle delirium, and antiemetics.

Data on 1 sedated PGL patient and 2 patients in the placebo group were excluded after their identification, and none required active intervention. There was another patient who complained of blurred vision: de-coding revealed that he belonged to the placebo group. Two other PGL patients reported a brief episode of dizziness; the drug was immediately stopped and the dizziness disappeared without further harm. It can be deduced that PGL at the given regimen, besides its pain-associated benefits, did not impair intra- and postsurgery clinical signs and symptoms, and no severe adverse events were attributed to it.

The duration of the beneficial effects of PGL does not seem to depend exclusively on the duration of its administration. There is general consensus that even if PGL is administered for 5 or more days postoperatively, its benefits are apparent for only 24 to 48 hours. ${ }^{8,10,22,33}$ We administered PGL for 3 days instead of 7 days, as described by others ${ }^{23}$ because of the known limited time effect of the drug, $8,10,22,33$ the consideration that brain interventions are less painful than, for example, orthopedic interventions, ${ }^{10}$ and to minimize the occurrence of potential side effects. Further studies are warranted to evaluate the long-term effects of PGL in neurosurgical patients.

The lower number of PGL patients using analgesics at 1 month following surgery and their overall $25 \%$ to $40 \%$ better pain scores in comparison with placebo-administered matched controls further support PGL's beneficial analgesic effects. Some reports demonstrated late favorable PGL effects on evolving chronic pain, ${ }^{30,33}$ but others refuted those findings. ${ }^{22}$ Some reports also linked the analgesic effect to the duration of the administration of the drug (up to 14 days). ${ }^{10,22,26}$ Importantly, there was no case of hyperalgesia reported in either of our 2 groups. Nevertheless, since our protocol was designed to focus on acute postoperative pain and analgesic usage, the association between PGL and the reduction in long-term postoperative pain and analgesic use needs to be further investigated in depth.

A review of 55 nonneurosurgical studies ${ }^{33}$ appeared in the literature while we were finalizing this report. Similar to our findings, the authors concluded that twice-daily 75 to $150 \mathrm{mg}$ PGL improved at-rest and movement pain scores, spared opioid usage by approximately $28 \%$ at 24 hours, and reduced the PONV and pruritus rates. However, all of those benefits were at the expense of increased rates of sedation, dizziness, and visual disturbance, none of which occurred in our experience. The patients' acute pain outcomes were similar after any dose of PGL ranging between 100 to $300 \mathrm{mg}$ PGL that was given either as a single preoperative dose or multiple postoperative doses. ${ }^{33}$

\section{Limitations}

This study and the reported results bear several clinical limitations, all originating from the specific constituents of the protocol. First, the findings may not be valid for pa- 
tients undergoing urgent or emergent interventions or children who undergo craniotomy. Additional investigations are needed to elaborate the usefulness of the drug and the times and doses that are thereby appropriate. In addition, since patients in other institutions may not be admitted the night before surgery, they may be given the study medication to be taken at home on the evening before surgery. Third, this study reports data concerning an extremely small number of patients who underwent infratentorial interventions mixed with supratentorial ones. Some would allege that pain in infratentorial surgery is more intense than that in supratentorial surgery. Nevertheless, pain in the former would originate mostly from the neck muscles that are injured during surgery or during intervention that involves very low anatomical areas such as $\mathrm{C} 4-5$. None of our patients had such surgery; therefore, pain would not appear different in the 2 subgroups. The same could be said for the very small and identical number of patients who were included in the study while undergoing awake craniotomy. Nonawake craniotomies were performed in the vast majority of patients (84\%), so the impact of the "awake" group on pain control versus general anesthesia is clinically nil. This is besides the fact that the brain is non-nociceptive, and the non-brain tissue components of the cranium are equally painful in either group.

\section{Conclusions}

This study contributes to the expanding knowledge on the perioperative administration of twice-daily $150 \mathrm{mg}$ PGL. Primarily, its prolonged perioperative administration did not interfere with patient safety and outcomes after intracranial surgery. We demonstrated that 1 dose of PGL improved the quality of sleep during the night before elective craniotomy, while 2 doses significantly alleviated the patients' presurgical anxiety. The addition of $150 \mathrm{mg}$ twice-daily PGL for 72 hours after surgery to commonly used analgesics yielded better pain scores in comparison with placebo and concomitantly reduced the doses and consumption rates of both analgesics and the need for antiemetics. There were no significant adverse effects or unacceptably excessive sedation levels associated with PGL, which seemingly contributed to the observed reduction in PONV.

\section{Acknowledgments}

Esther Eshkol, Scientific and Linguistic Editor at the Tel Aviv Sourasky Medical Center, is thanked for editorial assistance; Tal Bdolah-Abram, MA, statistician at the Hadassah Hebrew University, Jerusalem, is thanked for the statistical analyses; and Meirav Yehuda, Medical Pharmacist at the Tel Aviv Sourasky Medical Center, is thanked for her assistance in supervising the randomization and blinding protocol.

\section{References}

1. Baidya DK, Agarwal A, Khanna P, Arora MK: Pregabalin in acute and chronic pain. J Anaesthesiol Clin Pharmacol 27:307-314, 2011

2. Batoz H, Verdonck O, Pellerin C, Roux G, Maurette P: The analgesic properties of scalp infiltrations with ropivacaine after intracranial tumoral resection. Anesth Analg 109:240 244, 2009

3. Ben-Menachem E: Pregabalin pharmacology and its rel- evance to clinical practice. Epilepsia 45 (Suppl 6):13-18, 2004

4. Bonnet F, Marret E: Postoperative pain management and outcome after surgery. Best Pract Res Clin Anaesthesiol 21:99-107, 2007

5. Breivik H: Postoperative pain management: why is it difficult to show that it improves outcome? Eur J Anaesthesiol 15:748-751, 1998

6. Breivik H, Stubhaug A: Management of acute postoperative pain: still a long way to go! Pain 137:233-234, 2008

7. Brown AK, Christo PJ, Wu CL: Strategies for postoperative pain management. Best Pract Res Clin Anaesthesiol 18:703-717, 2004

8. Burke SM, Shorten GD: Perioperative pregabalin improves pain and functional outcomes 3 months after lumbar discectomy. Anesth Analg 110:1180-1185, 2010

9. Buvanendran A, Kroin JS: Useful adjuvants for postoperative pain management. Best Pract Res Clin Anaesthesiol 21:31-49, 2007

10. Buvanendran A, Kroin JS, Della Valle CJ, Kari M, Moric M, Tuman KJ: Perioperative oral pregabalin reduces chronic pain after total knee arthroplasty: a prospective, randomized, controlled trial. Anesth Analg 110:199-207, 2010

11. Buvanendran A, Kroin JS, Kari M, Tuman KJ: Can a single dose of $300 \mathrm{mg}$ of pregabalin reach acute antihyperalgesic levels in the central nervous system? Reg Anesth Pain Med 35:535-538, 2010

12. Carr DB, Goudas LC: Acute pain. Lancet 353:2051-2058, 1999

13. Carstensen M, Møller AM: Adding ketamine to morphine for intravenous patient-controlled analgesia for acute postoperative pain: a qualitative review of randomized trials. Br J Anaesth 104:401-406, 2010

14. Chelly JE, Ploskanych T, Dai F, Nelson JB: Multimodal analgesic approach incorporating paravertebral blocks for open radical retropubic prostatectomy: a randomized double-blind placebo-controlled study. Can J Anaesth 58:371-378, 2011

15. Cheung CW, Choi WS, Leung YY, Lui F, Ng JK, Hei-Ho AM, et al: A double-blind randomized crossover study to evaluate the timing of pregabalin for third molar surgery under local anesthesia. J Oral Maxillofac Surg 70:25-30, 2012

16. Cold GE, Felding M: Even small doses of morphine might provoke "luxury perfusion" in the postoperative period after craniotomy. Neurosurgery 32:327, 1993

17. Dauri M, Faria S, Gatti A, Celidonio L, Carpenedo R, Sabato AF: Gabapentin and pregabalin for the acute post-operative pain management. A systematic-narrative review of the recent clinical evidences. Curr Drug Targets 10:716-733, 2009

18. Dworkin RH, Corbin AE, Young JP Jr, Sharma U, LaMoreaux L, Bockbrader H, et al: Pregabalin for the treatment of postherpetic neuralgia: a randomized, placebo-controlled trial. Neurology 60:1274-1283, 2003

19. Eipe N, Penning J, Yazdi F, Mallick R, Turner L, Ahmadzai $\mathrm{N}$, et al: Perioperative use of pregabalin for acute pain-a systematic review and meta-analysis. Pain 156:1284-1300, 2015

20. Ekstein MP, Weinbroum AA: Immediate postoperative pain in orthopedic patients is more intense and requires more analgesia than in post-laparotomy patients. Pain Med 12:308313, 2011

21. Engelman E, Cateloy F: Efficacy and safety of perioperative pregabalin for post-operative pain: a meta-analysis of randomized-controlled trials. Acta Anaesthesiol Scand 55:927-943, 2011

22. Fassoulaki A, Melemeni A, Tsaroucha A, Paraskeva A: Perioperative pregabalin for acute and chronic pain after abdominal hysterectomy or myomectomy: a randomised controlled trial. Eur J Anaesthesiol 29:531-536, 2012 
23. Freedman BM, O'Hara E: Pregabalin has opioid-sparing effects following augmentation mammaplasty. Aesthet Surg J 28:421-424, 2008

24. Hartrick C, Van Hove I, Stegmann JU, Oh C, Upmalis D: Efficacy and tolerability of tapentadol immediate release and oxycodone $\mathrm{HCl}$ immediate release in patients awaiting primary joint replacement surgery for end-stage joint disease: a 10-day, phase III, randomized, double-blind, active- and placebo-controlled study. Clin Ther 31:260-271, 2009

25. Hurley RW, Cohen SP, Williams KA, Rowlingson AJ, Wu CL: The analgesic effects of perioperative gabapentin on postoperative pain: a meta-analysis. Reg Anesth Pain Med 31:237-247, 2006

26. Jensen MP, Hsu PH, Vanhove GF: Early pain reduction can predict treatment response: results of integrated efficacy analyses of a once-daily gastroretentive formulation of gabapentin in patients with postherpetic neuralgia. Pain Med 13:1059-1066, 2012

27. Joshi GP: Multimodal analgesia techniques and postoperative rehabilitation. Anesthesiol Clin North America 23:185202, 2005

28. Kehlet H, Dahl JB: The value of "multimodal" or "balanced analgesia" in postoperative pain treatment. Anesth Analg 77:1048-1056, 1993

29. Kollender Y, Bickels J, Stocki D, Maruoani N, Chazan S, Nirkin A, et al: Subanaesthetic ketamine spares postoperative morphine and controls pain better than standard morphine does alone in orthopaedic-oncological patients. Eur J Cancer 44:954-962, 2008

30. Lavand'homme P: The progression from acute to chronic pain. Curr Opin Anaesthesiol 24:545-550, 2011

31. Lesser H, Sharma U, LaMoreaux L, Poole RM: Pregabalin relieves symptoms of painful diabetic neuropathy: a randomized controlled trial. Neurology 63:2104-2110, 2004

32. Mercadante S, Caraceni A: Conversion ratios for opioid switching in the treatment of cancer pain: a systematic review. Palliat Med 25:504-515, 2011

33. Mishriky BM, Waldron NH, Habib AS: Impact of pregabalin on acute and persistent postoperative pain: a systematic review and meta-analysis. Br J Anaesth 114:10-31, 2015

34. Moore RA, Straube S, Wiffen PJ, Derry S, McQuay HJ: Pregabalin for acute and chronic pain in adults. Cochrane Database Syst Rev (3):CD007076, 2009

35. Nesher N, Ekstein MP, Paz Y, Marouani N, Chazan S, Weinbroum AA: Morphine with adjuvant ketamine vs higher dose of morphine alone for immediate postthoracotomy analgesia. Chest 136:245-252, 2009

36. Pesonen A, Suojaranta-Ylinen R, Hammarén E, Kontinen VK, Raivio P, Tarkkila P, et al: Pregabalin has an opioidsparing effect in elderly patients after cardiac surgery: a randomized placebo-controlled trial. Br J Anaesth 106:873881,2011

37. Rakhman E, Shmain D, White I, Ekstein MP, Kollender Y, Chazan S, et al: Repeated and escalating preoperative subanesthetic doses of ketamine for postoperative pain control in patients undergoing tumor resection: a randomized, placebocontrolled, double-blind trial. Clin Ther 33:863-873, 2011

38. Randinitis EJ, Posvar EL, Alvey CW, Sedman AJ, Cook JA, Bockbrader HN: Pharmacokinetics of pregabalin in subjects with various degrees of renal function. J Clin Pharmacol 43:277-283, 2003
39. Ryzenman JM, Pensak ML, Tew JM Jr: Headache: a quality of life analysis in a cohort of 1,657 patients undergoing acoustic neuroma surgery, results from the acoustic neuroma association. Laryngoscope 115:703-711, 2005

40. Sylvester RK, Lindsay SM, Schauer C: The conversion challenge: from intrathecal to oral morphine. Am J Hosp Palliat Care 21:143-147, 2004

41. Syrmis W, Good P, Wootton J, Spurling G: Opioid conversion ratios used in palliative care: is there an Australian consensus? Intern Med J 44:483-489, 2014

42. Tuchman M, Barrett JA, Donevan S, Hedberg TG, Taylor CP: Central sensitization and $\mathrm{Ca}(\mathrm{V}) \alpha_{2} \delta$ ligands in chronic pain syndromes: pathologic processes and pharmacologic effect. J Pain 11:1241-1249, 2010

43. Türe H, Sayin M, Karlikaya G, Bingol CA, Aykac B, Türe U: The analgesic effect of gabapentin as a prophylactic anticonvulsant drug on postcraniotomy pain: a prospective randomized study. Anesth Analg 109:1625-1631, 2009

44. Vijayan N: Postoperative headache in acoustic neuroma. Headache 35: $98-100,1995$

45. Weinbroum AA: Non-opioid IV adjuvants in the perioperative period: pharmacological and clinical aspects of ketamine and gabapentinoids. Pharmacol Res 65:411-429, 2012

46. Weinbroum AA: Superiority of postoperative epidural over intravenous patient-controlled analgesia in orthopedic oncologic patients. Surgery 138:869-876, 2005

47. White PF, Tufanogullari B, Taylor J, Klein K: The effect of pregabalin on preoperative anxiety and sedation levels: a dose-ranging study. Anesth Analg 108:1140-1145, 2009

48. Wood S: Postoperative pain 1: understanding the factors affecting patients' experiences of pain. Nurs Times 106:10-13, 2010

49. Wu CL, Raja SN: Treatment of acute postoperative pain. Lancet 377:2215-2225, 2011

\section{Disclosures}

The authors report no conflict of interest concerning the materials or methods used in this study or the findings specified in this paper.

\section{Author Contributions}

Conception and design: Weinbroum, Shimony, Amit, Minz, Gonen. Acquisition of data: Weinbroum, Shimony, Amit, Minz, Kandov. Analysis and interpretation of data: Weinbroum, Shimony, Amit, Dany. Drafting the article: Weinbroum, Shimony, Amit, Gonen. Critically revising the article: Weinbroum, Shimony, Amit, Minz, Grossman, Ram. Reviewed submitted version of manuscript: Weinbroum, Shimony, Amit, Grossman, Kandov, Ram. Approved the final version of the manuscript on behalf of all authors: Weinbroum. Statistical analysis: Weinbroum, Shimony, Amit. Administrative/technical/material support: Weinbroum, Shimony, Amit. Study supervision: Weinbroum, Shimony, Amit, Ram.

\section{Correspondence}

Avi A. Weinbroum, Department of Anesthesiology and CCM, Tel Aviv Sourasky Medical Center, 6 Weizman St., Tel Aviv 6423906, Israel.email: aviw@tlvmc.gov.il. 